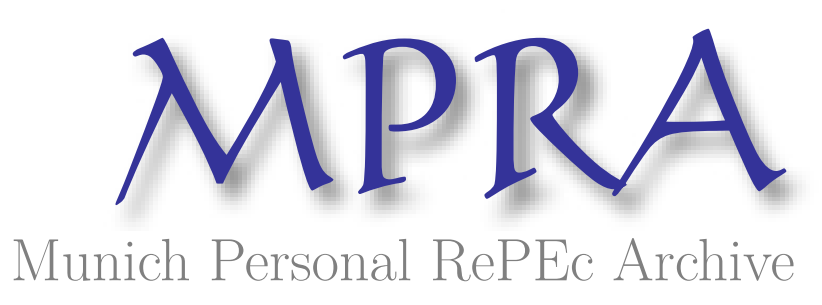

\title{
A Fast and Parsimonious Way to Estimate the Implied Rate of Return of Equity
}

Sanna, Dario

1 July 2020

Online at https://mpra.ub.uni-muenchen.de/102003/

MPRA Paper No. 102003, posted 23 Jul 2020 02:02 UTC 
A fast and parsimonious way to estimate the implied rate of return of equity

Dario Sanna

July 2020

codex.sanna.dario@gmail.com 
A fast and parsimonious way to estimate the implied rate of return of equity

\begin{abstract}
I propose a fast and parsimonious way to estimate the implied rate of return of common equity of single stocks and indexes, resulting from the combination of two easily computable ratios.
\end{abstract}

KEYWORDS: Earnings Yield, Implied Cost of Equity, Price Earnings Ratio, Quadratic Roe Ratio, Roe Discount Model 
I propose a fast and parsimonious way to estimate the implied rate of return of common equity of single stocks and indexes, resulting from the combination of two easily computable ratios.

The first ratio is the (EY) Earnings Yield rate, a well known and used measure of return, the second ratio is a brand new one, the QRR (Quadratic Roe Ratio) that, luckily, is just as easy to compute that the first ratio.

\section{The first part of the formula: the Equity Yield}

The inverse of the Price Earnings ratio, the Equity Yield rate, is a direct measure of return to the shareholder, expressed in percentage form and so directly comparable with bond return.

$K e=\frac{E p s_{1}}{P_{0}}(1)$

Where Eps ${ }_{1}$ is the Earnings per share forecasted on the next 12 months and $\mathrm{P}_{0}$ is the current price of the share.

Unfortunately, when the payout is lower than $100 \%$, the formula produces a downward biased estimate. Its value is at the low end of the range of plausible estimates.

\section{The second part of the formula: the QRR rate}

The QRR (Quadratic Roe Ratio) is a new, fast and easy, equity valuation formula, based on a single parameter: the Roe.

The formula is extremely simple: $P B=\frac{R o e^{2}}{K e^{2}}$

Where $\mathrm{PB}$ is the ratio of $\mathrm{P}_{0}$, the current price of the share, divided by $\mathrm{Bps}_{0}$, the latest book value of the share available. Roe is the ratio of the net income of the firm (trailing or forecasted) divided by the shareholders' equity. Ke is the implied market return.

From the (2) formula we can directly compute the value of Ke: 
$K e=\frac{R o e}{\sqrt{P B}}(3)$

The (3) formula is an indirect derivation of the Easton (2003) formula:

$$
K e=\sqrt{\frac{E p s_{2}-E p s_{1}+K e * D p s_{1}}{P_{0}}}
$$

where we impose Payout $=0$ and a constant return of equity during the next 12 and 24 months, with $\operatorname{Roe}_{1}=$ Roe $_{2}$.

QRR computation doesn't make use of dividends, buybacks or growth estimates.

QRR has been developed for a correct valuation of not dividend paying stocks.

Unfortunately, when used to evaluate dividend paying companies, it produces upward biased results. Its value is at the high end of the range of plausible estimates.

\section{The combined formula: the RDM}

So we have a good formula to evaluate $100 \%$ paying out shares, and another that's good for evaluating zero dividend paying firms.

I propose a way to get the right valuation formula starting from these two flawed ones, overcoming the limitation of the two components

The solution is straight and consists of simply combining both of them with a weighting equal to the actual payout rate of the stocks we are trying to evaluate (and portfolios and indexes as well).

I name this blended valuation as Roe Discount Model.

The RDM formula is the following:

$K e=\frac{R o e}{P B} * P Y+\frac{R o e}{\sqrt{P B}} *(1-P Y)$

Where the first half expresses the return of the dividend stream and the second half the return of the growth component.

PY is the current payout of earnings, in percent. 
An alternative formulation, using Eps, Dps and Bps instead of Roe and PB is the following:

$K e=\frac{\frac{E p s_{1}}{B p s_{0}}}{\frac{P_{0}}{B p s_{0}}} * P Y+\frac{\frac{E p s_{1}}{B p s_{0}}}{\sqrt{\frac{P_{0}}{B p s_{0}}}} *(1-P Y)$

equivalent to the simpler:

$K e=\frac{E p s_{1}}{P_{0}} * P Y+\frac{E p s_{1}}{\sqrt{P_{0} * B p s_{0}}} *(1-P Y)$

\section{The rationale of mixing valuation methods}

Since each company is free to choose its own payment policy, I assume two extreme scenarios.

In the first case the firm pays out all the earned profit as dividends.

In the second case the firm keeps all the money inside.

As we have a good way to calculate the implied return of these special cases, we can estimate the implied return of the whole firm by taking the (weighted) average of these two extreme returns.

\section{Limitations and defects}

The Roe Discount Model requires that you insert the forecast of the value of two parameters into the formula (Roe and Payout or Eps and Dps, depending to the chosen version), so which number are we going to use?

Differently from the many other more data-hungry valuation methods, that require the estimate and forecast of many data on a multi-year horizon, we just need two figures.

It is very similar to the single figure required for the most used valuation method: the PE. The problem is: which number are we going to use? 
It is the same problem that you run into with the data to put into the Terminal Value estimate when doing a DCF valuations, but applied now and not 5 years from now.

By the way, RDM looks particularly valuable in this case, since the standard formula

$T V=\frac{E_{n}+g}{K e-g}$

is quite a lot sensitive to growth rate assumptions.

Ideal solution: we should get and use an estimate of the most probable foreseeable future for the firm, the sector and the economy.

Good solution: next year forecast, adjusted for analysts' optimistic bias and sector cyclically profitability.

Just acceptable solution: trailing actual data of the latest 4 quarters, smoothed and corrected for non recurring financial items.

You can not escape it, when you use a thrifty formula, such as the one proposed here (or the PE ratio), what you gain with a very simple mathematics, you pay with a high sensitivity to the chosen value.

\section{Some few data to check the numbers}

To check the plausibility of the numbers coming out from the RDM, I paired its estimates to the estimates published by Aswath Damodaran, Professor of Finance at the Stern School of Business at New York University.

The sample is formed by 41 observations of trailing Eps and Dps of the 500 S\&P index, covering a horizon starting January 1, 2010 and ending January 1, 2020.

The data are exposed in Table 1 and Figure 1.

The mean (median) implied cost of equity estimated respectively by Damodaran and by the Roe Discount Model is 8,14\% (8,09\%) and 8,15\% (8,43\%). 
The standard deviation is respectively $0,51 \%$ and $1,61 \%$, with RDM showing a descending trend while Damodaran's estimates are not showing any discernible trend.

I also employed the Roe Discount Model to make a one time estimate of the implied cost of equity of the components of the Dow Jones index.

This time I used the analysts' forecasts, adjusted to a 12 months fixed horizon.

The data are exposed in Table 2.

The mean (median) implied cost of equity, estimated on 24 of the 30 companies included in the index, is $8,25 \%(8,41 \%)$.

For 6 firms I couldn't make any estimate as their book value was negative (Home Depot, McDonald's and Boeing) or the expected earnings are lower than dividends (Dow, Chevron and Exxon), preventing the use of the RDM formula.

I emphasize that since I used as input the consensus of analysts' forecasts, results are for sure higher than the correct ones.

Untabulated results show that, using as input the mean of trailing and forecasted 12 and 24 months data, there is a reduction of the dispersion of results vs using single year data.

\section{Summary and conclusion}

I describe a model able to obtain estimates of the cost of equity capital using as input only four parameters: current price, latest book value, forecast of earnings and dividends.

The model produces reasonable results, in line with third parts estimates.

I'm the first to admit that I have presented only an anecdotal prove of the utility of the new valuation formula, whose greatest merit is for now its simpleness.

A lot of statistical work has to be done to find out whether the estimates of the implied cost of equity produced by the Roe Discount Model are of some value.

We need to know how much its results are accurate, reliable and unbiased and may satisfy the need of scholars and practitioners as a tool to predict the future return of investments. 
Particularly we need to compare the actual return of RDM based portfolios vs more elaborated models as the dividend discount models, residual income valuation models, abnormal growth models or simple heuristic methods as the ranking by PE or PEG. 
References

Addis, David P., Growth Adjusted Price to Earnings Ratio and Implied Growth Rate (August 28, 2012). Available at SSRN: https://ssrn.com/abstract=2138483 or http://dx.doi.org/10.2139/ssrn.2138483

Alcock, Jamie and Mollee, Thomas and Wood, James, The Price of Earnings for Firms with Volatile Earnings Growth (May 13, 2008). Available at SSRN: https://ssrn.com/abstract=1133077 or http://dx.doi.org/10.2139/ssrn.1133077

Anderson, Keith P. and Brooks, Chris, Decomposing the Price-Earnings Ratio (May 2005). Available at SSRN: https://ssrn.com/abstract=739665 or http://dx.doi.org/10.2139/ssrn.739665

Asness, Cliff S., Fight the Fed Model: The Relationship between Stock Market Yields, Bond Market Yields, and Future Returns (December 2002). Available at SSRN: https://ssrn.com/abstract=381480 or http://dx.doi.org/10.2139/ssrn.381480

Bali, Turan G. and Demirtas, K. Ozgur and Tehranian, Hassan, Aggregate Earnings, Firm-Level Earnings and Expected Stock Returns (2008). Journal of Financial and Quantitative Analysis (JFQA), Vol. 43, No. 3, pp. 657-684, 2008. Available at SSRN: https://ssrn.com/abstract=1434355

Ball, Ray and Gerakos, Joseph and Linnainmaa, Juhani T. and Nikolaev, Valeri V., Earnings, Retained Earnings, and Book-to-Market in the Cross Section of Expected Returns (January 18, 2019). Journal of Financial Economics (JFE), Forthcoming. Available at SSRN: https://ssrn.com/abstract=2924798 or http://dx.doi.org/10.2139/ssrn.2924798

Bekaert, Geert and Ang, Andrew, Stock Return Predictability: Is it There? (April 2001). NBER Working Paper No. w8207. Available at SSRN:

https://ssrn.com/abstract $=265301$ 
Botosan, Christine and Plumlee, Marlene A., Estimating Expected Cost of Equity Capital: A Theory-Based Approach (July 2001). University of Utah Working Paper. Available at SSRN: https://ssrn.com/abstract=279309 or http://dx.doi.org/10.2139/ssrn.279309

Campbell, John Y. and Shiller, Robert J., Valuation Ratios and the Long-Run Stock Market Outlook: An Update (April 2001). NBER Working Paper No. w8221. Available at SSRN: https://ssrn.com/abstract=266191

Chan, Leo H., A More Intuitive Formula for PEG Ratio (November 2019). Available at SSRN: https://ssrn.com/abstract=3384411 or http://dx.doi.org/10.2139/ssrn.3384411

Chattopadhyay, Akash and Lyle, Matthew R. and Wang, Charles C. Y., Expected Stock Returns Worldwide: A Log-Linear Present-Value Approach (September 16, 2019). Harvard Business School Accounting \& Management Unit Working Paper No. 18-079. Available at SSRN: https://ssrn.com/abstract=3129086 or http://dx.doi.org/10.2139/ssrn.3129086

Chen, Peter Fusheng and Zhang, Guochang, How Do Accounting Variables Explain Stock Price Movements? Theory and Evidence (June 2006). Available at SSRN: https://ssrn.com/abstract=913463 or http://dx.doi.org/10.2139/ssrn.913463

Chiarella, Carl and Gao, Shenhuai, Solving the Price-Earnings Puzzle (April 2002). UTS Working Paper No. 116. Available at SSRN: https://ssrn.com/abstract=880002 or http://dx.doi.org/10.2139/ssrn.880002

Chin, Michael and Polk, Christopher, A Forecast Evaluation of Expected Equity Return Measures (January 16, 2015). Bank of England Working Paper No. 520. Available at SSRN: https://ssrn.com/abstract $=2550800$

Damodaran, Aswath, Equity Risk Premiums (ERP): Determinants, Estimation and Implications - The 2010 Edition (February 14, 2010). Available at SSRN: https://ssrn.com/abstract=1556382 or http://dx.doi.org/10.2139/ssrn.1556382 
DiGabriele, James A. and Eisner, Alan B, Putting the E Back in P/E Ratios. The Valuation Examiner, pp. 24-27, December 2003. Available at SSRN:

https://ssrn.com/abstract $=900626$

Dudney, Donna and Jirasakuldech, Benjamas and Zorn, Thomas S., P/E Movements: Some New Results (January 9, 2004). Available at SSRN: https://ssrn.com/abstract=499202 or http://dx.doi.org/10.2139/ssrn.499202

Easton, Peter D., Does the Peg Ratio Rank Stocks According to the Market's Expected Rate of Return on Equity Capital? (January 2002). Ohio State University Working Paper. Available at SSRN: https://ssrn.com/abstract $=301837$ or http://dx.doi.org/10.2139/ssrn.301837

Easton, Peter D., Pe Ratios, Peg Ratios, and Estimating the Implied Expected Rate of Return on Equity Capital (July 2003). Available at SSRN: https://ssrn.com/abstract $=423601$ or http://dx.doi.org/10.2139/ssrn.423601

Estrada, Javier, Adjusting P/E Ratios by Growth and Risk: The Perg Ratio. EFMA 2003 Helsinki Meetings. Available at SSRN: https://ssrn.com/abstract=389340 or http://dx.doi.org/10.2139/ssrn.389340

Ferguson, Robert, Making the Dividend Discount Model Relevant for Financial Analysts (October 26, 1998). Available at SSRN: https://ssrn.com/abstract=3104851 or http://dx.doi.org/10.2139/ssrn.3104851

Fitzgerald, Tristan and Gray, Stephen and Hall, Jason L. and Jeyaraj, Ravi, Unconstrained Estimates of the Equity Risk Premium (January 29, 2013). Forthcoming in Review of Accounting Studies, 2013, 18 (2), June, Accepted 26 March 2012.. Available at SSRN: https://ssrn.com/abstract=1551748 or http://dx.doi.org/10.2139/ssrn.1551748

Gebhardt, William R. and Lee, Charles M.C. and Swaminathan, Bhaskaran, Toward an Implied Cost of Capital (October 27, 2000). Parker Center for Investment Research 
Working Paper. Available at SSRN: https://ssrn.com/abstract=254523 or http://dx.doi.org/10.2139/ssrn.254523

Gode, Dan and Ohlson, James A., P-E Multiples and Changing Interest Rates (July 2000). NYU Working Paper No. Dhananjay (Dan) K. Gode-02. Available at SSRN: https://ssrn.com/abstract $=1280700$

Gode, Dhananjay (Dan) K. and Mohanram, Partha S., What Affects the Implied Cost of Equity Capital? (February 3, 2001). Stern School of Business Working Paper. Available at SSRN: https://ssrn.com/abstract=258702 or http://dx.doi.org/10.2139/ssrn.258702

Goldberg, Eliezer, Are Reinvested Earnings a Substitute for Dividends? (August 27, 2018). Available at SSRN: https://ssrn.com/abstract $=3244048$ or http://dx.doi.org/10.2139/ssrn.3244048

Golob, John, A Pragmatist's Guide to Long-Run Equity Returns, Market Valuation, and the CAPE (October 10, 2014). Available at SSRN: https://ssrn.com/abstract=2508382 or http://dx.doi.org/10.2139/ssrn.2508382

Hansen, Charlotte Strunk and Tuypens, Bjorn, Examining the Statistical Properties of Financial Ratios (January 27, 2005). EFA 2005 Moscow Meetings Paper. Available at SSRN: https://ssrn.com/abstract=676832 or http://dx.doi.org/10.2139/ssrn.676832

Henschke, Stefan and Homburg, Carsten, Equity Valuation Using Multiples: Controlling for Differences Between Firms (May 15, 2009). Available at SSRN: https://ssrn.com/abstract=1270812 or http://dx.doi.org/10.2139/ssrn.1270812

Jewell, Jeffrey Jay and Mankin, Jeffrey A., What Is Your EPS? Issues in Computing and Interpreting Earnings Per Share (2016). Jewell, J. J. and Mankin, J. A. (2016). What is Your EPS? Issues in Computing and Interpreting Earnings Per Share. Academy of Accounting and Financial Studies Journal, 20(3), 48-61.. Available at SSRN: https://ssrn.com/abstract=2827796 or http://dx.doi.org/10.2139/ssrn.2827796 
Jiang, Xiaoquan and Kang, Qiang, Cross-Sectional PEG Ratios, Market Equity Premium, and Macroeconomic Activity (August 27, 2012). 25th Australasian Finance and Banking Conference 2012. Available at SSRN: https://ssrn.com/abstract=2137295 or http://dx.doi.org/10.2139/ssrn.2137295

Ju, Nengjiu and Bakshi, Gurdip S., Book Values, Earnings, and Market Valuations (November 2002). AFA 2003 Washington, DC Meetings. Available at SSRN: https://ssrn.com/abstract=342680 or http://dx.doi.org/10.2139/ssrn.342680

Keimling, Norbert, Predicting Stock Market Returns Using the Shiller CAPE — An Improvement Towards Traditional Value Indicators? (January 21, 2016). Available at SSRN: https://ssrn.com/abstract=2736423 or http://dx.doi.org/10.2139/ssrn.2736423

Krishnan, C. N. V. and Chen, Yifei, The Relationship between Dividend Payout and Price to Earnings (December 9, 2019). Available at SSRN: https://ssrn.com/abstract=3016040 or http://dx.doi.org/10.2139/ssrn.3016040

Lew, Sung Hee, Stock Returns with Four Different Valuation Ratios (October 30, 2016). Available at SSRN: https://ssrn.com/abstract $=2859566$ or http://dx.doi.org/10.2139/ssrn.2859566

Lleo, Sebastien and Ziemba, William T., Does the Bond-Stock Earning Yield Differential Model Predict Equity Market Corrections Better than High P/E Models? (May 4, 2015). Available at SSRN: https://ssrn.com/abstract $=2296836$ or http://dx.doi.org/10.2139/ssrn.2296836

Maio, Paulo F. and Xu, Danielle, Cash-Flow or Return Predictability at Long Horizons? The Case of Earnings Yield (June 2, 2020). Available at SSRN: https://ssrn.com/abstract=2170079 or http://dx.doi.org/10.2139/ssrn.2170079 Mangelmans, Job and Rijken, Herbert A., Implied Growth Horizons and the Cost of Equity (May 4, 2016). Available at SSRN: https://ssrn.com/abstract=2775303 or http://dx.doi.org/10.2139/ssrn.2775303 
Murugesan, Narayanaswamy, When Equity Returns Are Different from Discount Rates: Why PEG Based DCF Valuation is Flawed (June 18, 2013). Available at SSRN: https://ssrn.com/abstract=2281228 or http://dx.doi.org/10.2139/ssrn.2281228

Ohlson, James A. Earnings, Book Values, and Dividends in Equity Valuation (1995). Contemporary Accounting Research, 11: 661-687. doi:10.1111/j.1911-3846.1995.tb00461.x Penman, Stephen H., A Synthesis of Equity Valuation Techniques and the Terminal Value Calculation for the Dividend Discount Model (June 1997). Available at SSRN: https://ssrn.com/abstract=38720 or http://dx.doi.org/10.2139/ssrn.38720

Penman, Stephen H., Combining Earnings and Book Value in Equity Valuation (July 1997). Available at SSRN: https://ssrn.com/abstract $=38721$ or http://dx.doi.org/10.2139/ssrn.38721

Penman, Stephen H. and Zhang, Xiao-Jun, Modeling Sustainable Earnings and P/E Ratios with Financial Statement Analysis (June 1, 2002). Available at SSRN: https://ssrn.com/abstract=318967 or http://dx.doi.org/10.2139/ssrn.318967 Penman, Stephen H. and Zhu, Julie, Accounting-Based Estimates of the Cost of Capital: A Third Way (February 1, 2017). Available at SSRN: https://ssrn.com/abstract=3049245 or http://dx.doi.org/10.2139/ssrn.3049245

Ranka, Arpan, Forecasting Equity Market Returns Using Historical Price Earnings Multiples (December 21, 2012). XI Capital Markets Conference, 21-22 December 2012, Indian Institute of Capital Markets (UTIICM) . Available at SSRN: https://ssrn.com/abstract=2255323 or http://dx.doi.org/10.2139/ssrn.2255323

Reggiani, Francesco and Penman, Stephen H., Returns to Buying Earnings and Book Value: Accounting for Growth and Risk (December 1, 2009). Available at SSRN: https://ssrn.com/abstract=1536618 or http://dx.doi.org/10.2139/ssrn.1536618 
Staehle, Martin and Lampenius, Niklas, Are PE- and MB-Ratios Susceptible to Accruals, Growth, or Profitability? (June 1, 2010). Available at SSRN: https://ssrn.com/abstract=1542023 or http://dx.doi.org/10.2139/ssrn.1542023

Staehle, Martin and Lampenius, Niklas, What is Driving the Price-to-Earnings Ratio: The Effect of Conservative Accounting and Growth (March 25, 2013). Available at SSRN: https://ssrn.com/abstract=2239208 or http://dx.doi.org/10.2139/ssrn.2239208

Sum, Vichet, Stock Market Performance: Variance Decomposition of Price-Earnings Ratio, Dividend Yield and Tobin's Q (July 14, 2013). Journal of Financial Transformation, 39, 63-67. April, 2014. Available at SSRN: https://ssrn.com/abstract=2293532 or http://dx.doi.org/10.2139/ssrn.2293532

Taliento, Marco, Effects of Dividend Ratios and Other Fundamentals on Forward P/Es - Some Evidences from a Worldwide Cross-Sectional Multivariate Analysis (November 12, 2012). Proceedings of 19th International Business Research Conference 2012. Available at SSRN: https://ssrn.com/abstract=2174331 or http://dx.doi.org/10.2139/ssrn.2174331

Vila Wetherilt, Anne and Weeken, Olaf, Equity Valuation Measures: What Can They Tell Us?. Bank of England Quarterly Bulletin, Winter 2002. Available at SSRN: https://ssrn.com/abstract=708981

Wang, Pengguo, The Implied Risk Premium and Firm Risk Characteristics (January 28, 2013). Available at SSRN: https://ssrn.com/abstract $=2208369$ or http://dx.doi.org/10.2139/ssrn.2208369

Yee, Kenton K., Aggregation, Dividend Irrelevancy, and Earnings-Value Relations. Contemporary Accounting Research, Vol. 22, No. 2, pp. 453-480, Summer 2005. Available at SSRN: https://ssrn.com/abstract $=667781$ 
Table 1

S\&P500 Data and Implied Equity Returns

\begin{tabular}{|c|c|c|c|c|c|c|c|c|c|c|c|c|}
\hline Date & $\begin{array}{c}\text { S\&P500 } \\
\text { Price }\end{array}$ & \begin{tabular}{|c|} 
S\&P500 \\
Bps
\end{tabular} & $\begin{array}{c}\text { S\&P500 } \\
\text { Eps } \\
\text { Trailing } \\
\end{array}$ & $\begin{array}{c}\text { S\&PFЮO } \\
\text { Dps } \\
\text { Trailing } \\
\end{array}$ & ROE & PY & PB & $\begin{array}{c}\text { US 10Y } \\
\text { Treasury } \\
\text { Rate } \\
\end{array}$ & $E Y$ & QQRR & RDM & DKE \\
\hline $\operatorname{Jan} 1,2000$ & & 451,37 & & & & & & & & & & \\
\hline Aрг 1,2000 & & 449,43 & & & & & & & & & & \\
\hline Jul 1,2000 & & 488,09 & & & & & & & & & & \\
\hline Oct 1,2000 & & 498,43 & & & & & & & & & & \\
\hline $\operatorname{Jan} 1,2010$ & $1.123,58$ & 513,58 & 60,51 & 26,600 & $13,41 \%$ & $44,0 \%$ & 2,19 & $3,73 \%$ & $5,39 \%$ & $9,06 \%$ & $7,45 \%$ & $8,09 \%$ \\
\hline Aрг 1,2010 & $1.197,32$ & 531,35 & 72,18 & 25,80 & $16,06 \%$ & $35,7 \%$ & 2,25 & $3,85 \%$ & $6,03 \%$ & $10,70 \%$ & $9,03 \%$ & $8,01 \%$ \\
\hline Jul 1,2010 & $1.080,20$ & $542,00-$ & 79,33 & 25,92 & $16,25 \%$ & $32,7 \%$ & 1,99 & $3,01 \%$ & $7,34 \%$ & $11,51 \%$ & $10,15 \%$ & $8,11 \%$ \\
\hline Oct 1,2010 & $1.171,58$ & 568,35 & 84,34 & 26,24 & $16,92 \%$ & $31,1 \%$ & 2,06 & $2,54 \%$ & $7,20 \%$ & $11,79 \%$ & $10,36 \%$ & $7,85 \%$ \\
\hline $\operatorname{Jan} 1,2011$ & $1.283,02$ & 579,14 & 90,48 & 26,59 & $17,62 \%$ & $29,4 \%$ & 2,22 & $3,39 \%$ & $7,05 \%$ & $11,84 \%$ & $10,43 \%$ & $8,59 \%$ \\
\hline Apr 1, 2011 & $1.331,51$ & 594,05 & 93,29 & 26,88 & $17,56 \%$ & $28,8 \%$ & 2,24 & $3,45 \%$ & $7,01 \%$ & $11,73 \%$ & $10,37 \%$ & $8,76 \%$ \\
\hline Jul 1, 2011 & $1.325,19$ & 613,08 & 95,26 & 27,65 & $17,58 \%$ & $29,0 \%$ & 2,16 & $3,00 \%$ & $7,19 \%$ & $11,95 \%$ & $10,5 \%$ & $8,72 \%$ \\
\hline Oct 1,2011 & $1.207,22$ & 613,18 & 98,29 & 28,46 & $17,29 \%$ & $29,0 \%$ & 1,97 & $2,15 \%$ & $8,14 \%$ & $12,33 \%$ & $11,11 \%$ & $9,79 \%$ \\
\hline $\operatorname{Jan} 1,2012$ & $1.300,58$ & 613,14 & 99,19 & 30,02 & $17,13 \%$ & $30,3 \%$ & 2,12 & $1,97 \%$ & $7,63 \%$ & $11,76 \%$ & $10,51 \%$ & $9,29 \%$ \\
\hline Арг 1,2012 & $1.386,43$ & 633,04 & 99,36 & 30,57 & $16,73 \%$ & $30,8 \%$ & 2,19 & $2,05 \%$ & $7,17 \%$ & $11,30 \%$ & $10,03 \%$ & $8,68 \%$ \\
\hline Jul 1,2012 & $1.360,18$ & 639,45 & 98,23 & 31,64 & $16,02 \%$ & $32,2 \%$ & 2,13 & $1,53 \%$ & $7,22 \%$ & $10,99 \%$ & $9,77 \%$ & $8,12 \%$ \\
\hline Oct 1,2012 & \begin{tabular}{|l|}
$1.438,22$ \\
\end{tabular} & 662,33 & 96,24 & 32,78 & $15,70 \%$ & $34,1 \%$ & 2,17 & $1,75 \%$ & $6,69 \%$ & $10,65 \%$ & $9,30 \%$ & $8,10 \%$ \\
\hline $\operatorname{Jan} 1,2013$ & $1.480,40$ & 667,37 & 97,00 & 34,89 & $15,82 \%$ & $36,0 \%$ & 2,22 & $1,91 \%$ & $6,55 \%$ & $10,62 \%$ & $9,1 \% \%$ & $7,91 \%$ \\
\hline Aрг 1,2013 & $1.571,10$ & 679,09 & $\overline{97,00}$ & 35,37 & $15,32 \%$ & $36,5 \%$ & 2,31 & $1,76 \%$ & $6,17 \%$ & $10,07 \%$ & $865 \%$ & $7,44 \%$ \\
\hline Jul 1,2013 & $1.669,08$ & 679,15 & 100,26 & 36,53 & $15,68 \%$ & $36,4 \%$ & 2,46 & $2,58 \%$ & $6,01 \%$ & $10,00 \%$ & $8,55 \%$ & $8,31 \%$ \\
\hline Oct 1,2013 & $1.720,03$ & 693,22 & $10 \sqrt[3]{3} 33$ & 37,67 & $15,60 \%$ & $36,5 \%$ & 2,48 & $2,62 \%$ & $6,01 \%$ & $9,90 \%$ & $848 \%$ & 8,27 \\
\hline $\operatorname{Jan} 1,2014$ & $1.822,36$ & 716,24 & 110,24 & 38,50 & $16,52 \%$ & $34,9 \%$ & 2,54 & $2,86 \%$ & $6,05 \%$ & $10,36 \%$ & $8,85 \%$ & $7,82 \%$ \\
\hline Aрг 1,2014 & \begin{tabular}{|l|}
$1.864,26$ \\
\end{tabular} & 718,21 & 109,43 & 39,31 & $16,11 \%$ & $35,9 \%$ & 2,60 & $2,71 \%$ & $5,87 \%$ & $10,00 \%$ & $8,52 \%$ & $7,86 \%$ \\
\hline Jul 1,2014 & $1.973,10$ & 734,24 & 111,33 & 40,21 & $16,39 \%$ & $36,1 \%$ & 2,69 & $2,54 \%$ & $5,64 \%$ & $10,0 \% \%$ & $8,43 \%$ & $7,92 \%$ \\
\hline Oct 1,2014 & $1.937,27$ & 737,18 & 114,13 & 41,46 & $16,46 \%$ & $36,3 \%$ & 2,63 & $2,30 \%$ & $5,89 \%$ & $10,16 \%$ & $8,61 \%$ & $7,73 \%$ \\
\hline Jaח 1,2015 & \begin{tabular}{|l|}
$2.028,18$ \\
\end{tabular} & 727,36 & 112,11 & 43,07 & $15,65 \%$ & $38,4 \%$ & 2,79 & $1,88 \%$ & $5,53 \%$ & $9,37 \%$ & $7,90 \%$ & $7,66 \%$ \\
\hline Арг 1, 2015 & 2.094,86 & 729,29 & 108,17 & 44,31 & $15,06 \%$ & $41,0 \%$ & 2,87 & $1,93 \%$ & $5,16 \%$ & $8,89 \%$ & $7,36 \%$ & $7,79 \%$ \\
\hline Jul 1, 2015 & 2.094,14 & 736,30 & 102,37 & 44,85 & $13,94 \%$ & $43,8 \%$ & 2,84 & $2,32 \%$ & $4,89 \%$ & $8,27 \%$ & $6,79 \%$ & $8,13 \%$ \\
\hline Oct 1,2015 & $2.024,81$ & 745,08 & 98,09 & 45,81 & $13,31 \%$ & $46,7 \%$ & 2,72 & $2,07 \%$ & $4,84 \%$ & $8,07 \%$ & $6,56 \%$ & $8,70 \%$ \\
\hline $\operatorname{Jan} 1,2016$ & $1.918,60$ & 740,29 & 94,20 & 47,03 & $12,95 \%$ & $49,9 \%$ & 2,59 & $2,09 \%$ & $4,91 \%$ & $8,04 \%$ & $6,45 \%$ & $8,21 \%$ \\
\hline Aрг 1,2016 & $2.075,54$ & 753,17 & 93,07 & 47,24 & $12,76 \%$ & $50,8 \%$ & 2,76 & $1,81 \%$ & $4,48 \%$ & $7,69 \%$ & $6,0 \% \%$ & $8,05 \%$ \\
\hline Jul 1, 2016 & $2.148,90$ & 765,12 & $\overline{92,46}$ & 47,30 & $12,56 \%$ & $51,2 \%$ & 2,81 & $1,50 \%$ & $4,30 \%$ & $7,49 \%$ & $5,86 \%$ & $7,77 \%$ \\
\hline Oct 1,2016 & $2.143,02$ & 780,01 & 95,01 & 47,82 & $12,75 \%$ & $50,3 \%$ & 2,75 & $1,76 \%$ & $4,43 \%$ & $7,69 \%$ & $6,05 \%$ & $7,92 \%$ \\
\hline $\operatorname{Jan} 1,2017$ & \begin{tabular}{|l|}
$2.275,12$ \\
\end{tabular} & 769,38 & 100,41 & 48,53 & $13,56 \%$ & $48,3 \%$ & 2,96 & $2,43 \%$ & $4,41 \%$ & $7,89 \%$ & $6,21 \%$ & $8,12 \%$ \\
\hline Арг 1,2017 & $2.359,31$ & 785,18 & 105,47 & 48,78 & $14,0 \mathrm{H} \%$ & $46,3 \%$ & 3,000 & $2,18 \%$ & $4,47 \%$ & $8,08 \%$ & $6,41 \%$ & $7,56 \%$ \\
\hline Jul 1,2017 & $2.454,10$ & 799,04 & 109,28 & 49,42 & $14,28 \%$ & $45,2 \%$ & 3,07 & $2,32 \%$ & $4,45 \%$ & $8,15 \%$ & $6,45 \%$ & $7,45 \%$ \\
\hline Oct 1,2017 & $2.557,0 \mathrm{OH}$ & 818,22 & 111,23 & 50,04 & $14,26 \%$ & $45,0 \%$ & 3,13 & $2,36 \%$ & $4,35 \%$ & $8,07 \%$ & $6,39 \%$ & $7,28 \%$ \\
\hline $\operatorname{Jan} 1,2018$ & \begin{tabular}{|l|}
$2.789,80$ \\
\end{tabular} & 826,52 & 114,28 & 50,89 & $14,85 \%$ & $44,5 \%$ & 3,38 & $2,58 \%$ & $4,10 \%$ & $8,08 \%$ & $6,31 \%$ & $7,66 \%$ \\
\hline Aрг 1, 2018 & $2.653,63$ & 833,44 & 119,00 & 51,37 & $15,16 \%$ & $43,2 \%$ & 3,18 & $2,87 \%$ & $4,48 \%$ & $8,49 \%$ & $6,76 \%$ & $8,06 \%$ \\
\hline Jul 1,2018 & $2.793,64$ & 836,39 & 125,02 & 51,88 & $15,65 \%$ & $41,5 \%$ & 3,34 & $2,89 \%$ & $4,48 \%$ & $8,56 \%$ & $6,87 \%$ & $8,26 \%$ \\
\hline Oct 1,2018 & \begin{tabular}{|l|}
$2.785,46$ \\
\end{tabular} & 843,58 & 132,43 & 53,16 & $16,19 \%$ & $40,1 \%$ & 3,30 & $3,15 \%$ & $4,75 \%$ & $8,91 \%$ & $7,24 \%$ & $8,53 \%$ \\
\hline $\operatorname{Jan} 1,2019$ & $2.607,39$ & 852,02 & 135,11 & 54,85 & $16,35 \%$ & $40,6 \%$ & 3,06 & $2,71 \%$ & $5,18 \%$ & $9,34 \%$ & $7,65 \%$ & $8,67 \%$ \\
\hline Арг 1, 2019 & $2.903,80$ & 868,42 & 135,55 & 55,41 & $16,26 \%$ & $40,9 \%$ & 3,34 & $2,53 \%$ & $4,67 \%$ & $8,89 \%$ & $7,17 \%$ & $8,28 \%$ \\
\hline Jul 1, 2019 & $2.996,11$ & 885,14 & 135,40 & 56,13 & $16,19 \%$ & $41,5 \%$ & 3,38 & $2,060 \%$ & $4,52 \%$ & $8,80 \%$ & $7,02 \%$ & $7,73 \%$ \\
\hline Oct 1,2019 & $2.977,68$ & 903,34 & 133,11 & 57,14 & $15,78 \%$ & $42,9 \%$ & 3,30 & $1,71 \%$ & $4,47 \%$ & $8,69 \%$ & $6,85 \%$ & $7,26 \%$ \\
\hline $\operatorname{Jan} 1,2020$ & \begin{tabular}{|l|}
$3.278,20$ \\
\end{tabular} & 914,49 & 139,15 & 58,40 & $16,33 \%$ & $42,0 \%$ & 3,58 & $1,76 \%$ & $4,24 \%$ & $8,63 \%$ & $6,79 \%$ & $6,96 \%$ \\
\hline 18 & & & & & & 3650 & & & & & & \\
\hline Mean & & & & & $15,39 \%$ & $39,0 \%$ & 2,60 & $2,45 \%$ & $5,72 \%$ & $9,67 \%$ & $815 \%$ & $8,14 \%$ \\
\hline Std. Dev. & & & & & $1,48 \%$ & $6,9 \%$ & 0,41 & $0,59 \%$ & $1,15 \%$ & $1,48 \%$ & $1,61 \%$ & $0,51 \%$ \\
\hline
\end{tabular}

EY: Earnings Yield, QRR: Quadratic Roe Return, RDM: Roe Discount Model,

DKE: Damodaran's Equity Cost (Trailing 12 m Equity Risk Premium + US 10Y Treasury rate);

Source 1: https://www.multpl.com/s-p-500-pe-ratio

Source 2: http://www.stern.nyu.edu/ adamodar/pc/implprem/ERPbymonth.xlsx 
Table 2

Dow Jones Components, Implied Equity Returns at 19 Jun 2020

\begin{tabular}{|c|c|c|c|c|c|c|c|c|c|c|c|}
\hline D.J & 18. Јип. 2020 & Price & Bps 0 & Eps 12 & Dps 12 & RDE 12 & PY 12 & $\mathrm{~PB} 0$ & EY & QRR & RDM \\
\hline & & & & & & & & & & & \\
\hline A.APL & Appla & 340,72 & 16.31 & 14,12 & 3,38 & $86,60 \%$ & $24,0 \%$ & 21,44 & $4,04 \%$ & $18,70 \%$ & 15,184 \\
\hline$A \times P$ & American Express & 100,84 & 27,01 & $4, \mathrm{~B} 5$ & $1,7 \mathrm{~B}$ & $17,85 \%$ & $36.8 \%$ & 3,74 & $4,80 \%$ & $8,28 \%$ & 7,649 \\
\hline$\overline{B A}$ & Boeing & 187.02 & $-8,01$ & 2,48 & 0.85 & & & & & & \\
\hline CAT & Caterpillar & $127,4 \mathrm{\theta}$ & 26.22 & 6.23 & 4,35 & $23,77 \%$ & $68.7 \%$ & 4,86 & $4,88 \%$ & $10.70 \%$ & 6,679 \\
\hline $\mathrm{Csco}$ & Cisco & 45,32 & B.47 & 2,67 & $1,4 \mathrm{~B}$ & $31,57 \%$ & $55.4 \%$ & 5,35 & $5,80 \%$ & $13,65 \%$ & $9,35 \%$ \\
\hline CVK & Cherron & 80.63 & 74,00 & 0,84 & 5,20 & & & & & & \\
\hline DIS & Walt Disney & 114,35 & $4 B, 87$ & 2,20 & 1,27 & $4,48 \%$ & $57.6 \%$ & 2,34 & $1,92 \%$ & $2,94 \%$ & $2,35 \%$ \\
\hline Dow & Dow & 41,65 & 17,32 & 1,53 & $2, \mathrm{Be}$ & & & & & & \\
\hline GS & Goldman Sachs & 201,63 & 225,05 & $16,8 \mathrm{~B}$ & 5,13 & $7.54 \%$ & $30.2 \%$ & 0,80 & $8,42 \%$ & $7.87 \%$ & $8,11 \%$ \\
\hline $\mathrm{HD}$ & Home Depot & 246.65 & $-2,20$ & 10,30 & 6,01 & & & & & & \\
\hline IBM & IBM & 122,47 & 25,55 & 8,62 & 6.50 & $37.66 \%$ & $67.5 \%$ & 4,70 & $7,86 \%$ & $17,20 \%$ & 10,8996 \\
\hline INTC & Intel & 58,62 & 18,75 & 4,60 & 1,36 & $24,55 \%$ & $28.6 \%$ & 3,18 & $7,72 \%$ & $13.77 \%$ & 11,989 \\
\hline JNJ & Johnson \& Johnson & 143,83 & 23,30 & 7.41 & 4,11 & $31,82 \%$ & $55,5 \%$ & 6,17 & $5,15 \%$ & $12,80 \%$ & $8,56 \%$ \\
\hline $\mathrm{JPM}$ & JPMorgan Chase & $87 . \mathrm{B} 1$ & 76,19 & $6,6 \mathrm{~B}$ & $3,6 \mathrm{~B}$ & B.77\% & $55.1 \%$ & $1,2 \mathrm{~B}$ & $6.83 \%$ & $7,74 \%$ & 7,249 \\
\hline KD & Coca-Cola & 46.02 & 4.27 & $1,9 \mathrm{~B}$ & 1,67 & $46.37 \%$ & $04,5 \%$ & 10.77 & $4,30 \%$ & $14,13 \%$ & 5,839 \\
\hline$M C D$ & McDonald's & 186.50 & $-11,65$ & 6.80 & 5,30 & & & & & & \\
\hline MMM & $3 M$ & $15 \mathrm{~B}, 15$ & 18,57 & 8,46 & 5,84 & $45,55 \%$ & $70.2 \%$ & 8,52 & $5,35 \%$ & $15,61 \%$ & $8,41 \%$ \\
\hline MRK & Merck & 77.80 & 10.71 & 4,62 & 2,47 & $43,18 \%$ & $53.5 \%$ & $7,2 \mathrm{~B}$ & $5.83 \%$ & $16,01 \%$ & $10,61 \%$ \\
\hline MSFT & Microsoft & 185.15 & 15,34 & 6.18 & 2,16 & $40,32 \%$ & $34,8 \%$ & 12,72 & $3,17 \%$ & $11,30 \%$ & $8,47 \%$ \\
\hline NKE & Nike & 85.70 & 5,56 & 2,21 & 0,84 & $39.71 \%$ & $42,8 \%$ & 17,24 & $2,30 \%$ & $8.56 \%$ & $6,46 \%$ \\
\hline PFE & Pfizer & 33,42 & $11, \mathrm{B2}$ & 2,06 & 1,54 & $17.42 \%$ & $74.7 \%$ & 2,83 & $6.16 \%$ & $10,36 \%$ & 7,229 \\
\hline$P G$ & Pixter \& Gamble & 118,92 & 18,10 & 5,15 & 3,20 & $26.89 \%$ & $62,2 \%$ & 6,23 & $4,33 \%$ & $10,82 \%$ & 6,799 \\
\hline RTX & Raytheon & $64, B 8$ & 52,81 & 2,71 & 2,01 & $5,14 \%$ & $74,0 \%$ & 1,23 & $4,18 \%$ & $4,63 \%$ & $4,309 \%$ \\
\hline TRV & Travelers & 116.50 & 102,00 & 8,57 & 3.47 & $8,38 \%$ & $36.3 \%$ & 1,14 & B. 21\% & $\mathrm{B}, 70 \%$ & $8,57 \%$ \\
\hline UNH & UnitedHealth & 281,24 & 64.76 & 16,32 & 4,76 & $25,21 \%$ & $28,1 \%$ & 4,50 & $5,61 \%$ & $11,88 \%$ & 10,069 \\
\hline V & Visa & 182,20 & 16.51 & 5,68 & 1,31 & $34,47 \%$ & $23,0 \%$ & 11,64 & $2,96 \%$ & $10,10 \%$ & $8,46 \%$ \\
\hline$V Z$ & Verizon & 56.10 & 15,55 & 4,66 & 2,51 & $20.89 \%$ & $53,8 \%$ & 3,61 & B, 30\% & $15,70 \%$ & 11,759 \\
\hline WBA & Walgreens Boots Alliance & 44,13 & 30,70 & 4,83 & 1,81 & $15,73 \%$ & $38,5 \%$ & 1,44 & $10,84 \%$ & $13,12 \%$ & $12,26 \%$ \\
\hline WMT & Walmart & 118,25 & 26,82 & 5.27 & 2,23 & $19,63 \%$ & $42.4 \%$ & 4,47 & $4,38 \%$ & $8,28 \%$ & 7,219 \\
\hline \multirow[t]{5}{*}{ KOM } & Exxon & $45.8 \mathrm{e}$ & 43,00 & 0,01 & 3,50 & & & & & & \\
\hline & & & & & & & & & & & \\
\hline & Median & & & & & $26,10 \%$ & $53.7 \%$ & 4,65 & $5,25 \%$ & $11,06 \%$ & 8,449 \\
\hline & Mean & & & & & $28,07 \%$ & $50.1 \%$ & 6.15 & $5,57 \%$ & $11,51 \%$ & $8,52 \%$ \\
\hline & Std. Dev. & & & & & $17.89 \%$ & $17.8 \%$ & 5,26 & $2,18 \%$ & $3,81 \%$ & $2,75 \%$ \\
\hline
\end{tabular}

Bps 0: Current Bps estimate, Eps 12: Forecasted Eps computed on a fixed 12 months horizon, Dps 12: Forecasted Dps computed on a fixed 12 months horizon, PY 12: Payout computed on a fixed 12 months horizon, ROE 12: Eps 12/ Bps 0, EY: Earnings Yield, QRR: Quadratic Roe Return, RDM: Roe Discount Model;

Source: https://www.marketscreener.com/ 
Figure 1

S\&P500 Estimates of Implied Cost of Equity

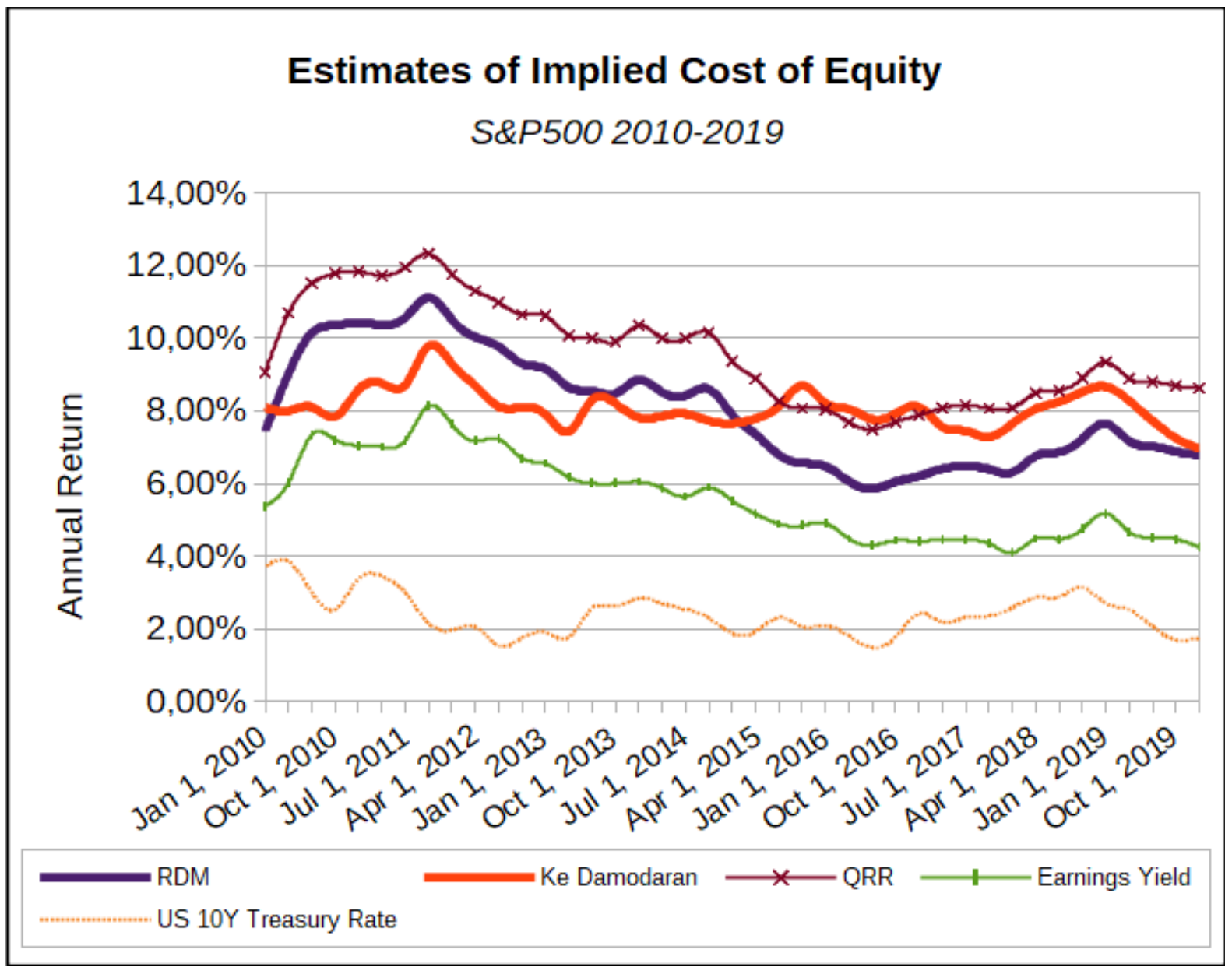

RDM: Roe Discount Model, Ke Damodaran: Damodaran's Equity cost (Trailing 12 month Equity Risk Premium + US 10Y Treasury rate), QRR: Quadratic Roe Return;

Source 1: https://www.multpl.com/s-p-500-pe-ratio

Source 2: http://www.stern.nyu.edu/ adamodar/pc/implprem/ERPbymonth.xlsx 